\title{
Cavity Processing Laboratory for SRF cavities
}

\section{F. Furuta, T. Ring}

Fermi National Accelerator Laboratory

FERMILAB-POSTER-20-010-TD

\section{Electrochemical Polishing (EP)}

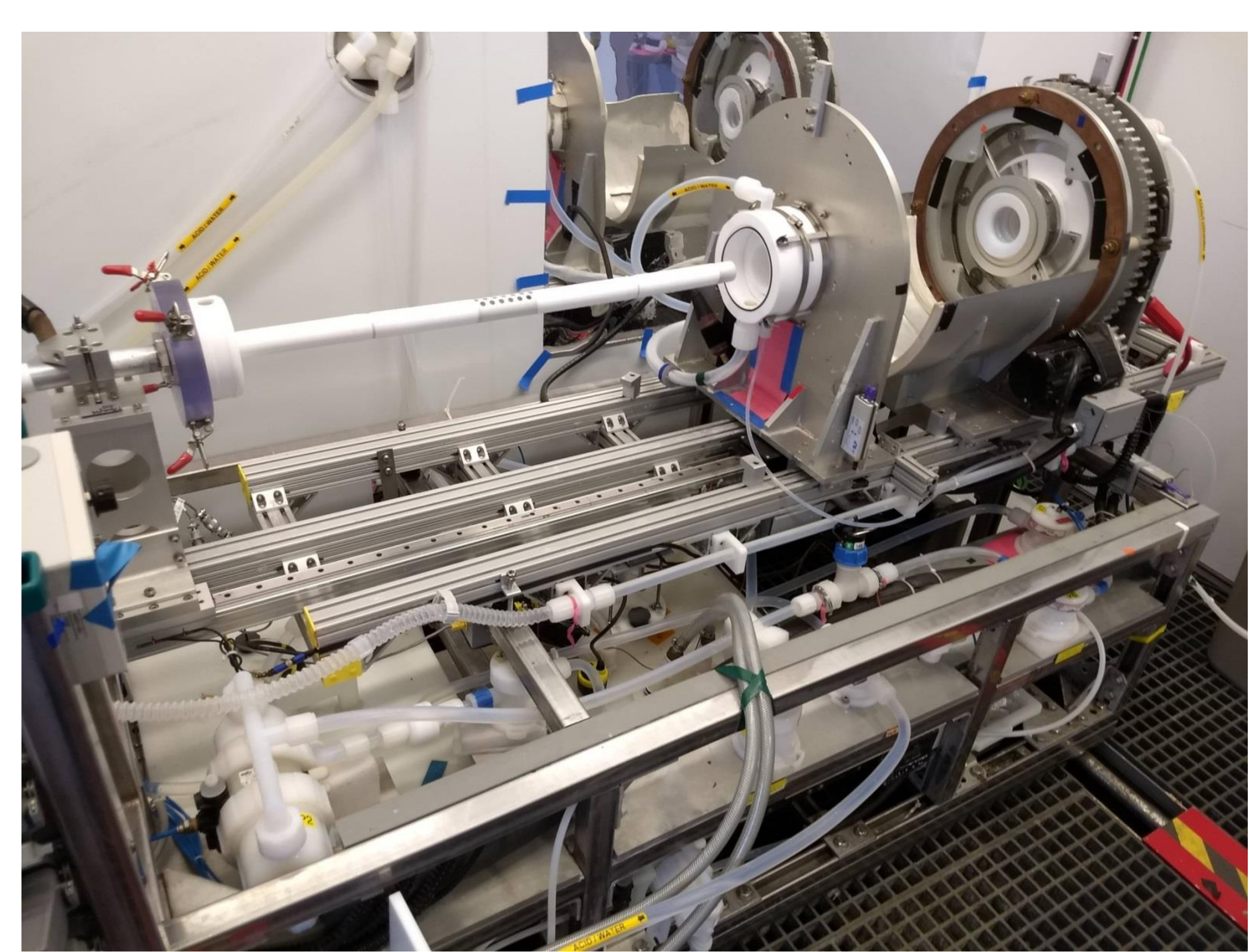

EP tool in IB4 CPL.

This tool can process a single-cell cavity with the frequency of $1.3 \mathrm{GHz}$ and higher. Multi-cell cavities and low frequency cavities are processed at ANL EP facility.
Electrochemical Polishing (EP) is an electrochemical process using an acid mixture (electrolyte) of sulfuric acid $\left(\mathrm{H}_{2} \mathrm{SO}_{4}, \sim 96 \%\right)$ and hydrofluoric acid (HF, $\sim 48 \%$ ) with the ratio of 10:1 in volume. EP electrolyte was pre-mixed by the company and delivered to Fermilab.

EP is performed by applying voltage between a cathode and an anode in an electrolyte bath. For the niobium cavities, aluminum (>99.5\%) is used as a cathode and niobium works as an anode.

The reaction can be described in two parts; 1 ) the electrochemical reaction develops the niobium pentoxide on the niobium surface, 2) the $\mathrm{HF}$ acid removes that developed oxide layer. These chemical reactions are written as:

$$
\begin{aligned}
& 2 \mathrm{Nb}+5 \mathrm{SO}_{4}{ }^{2-}+5 \mathrm{H}_{2} \mathrm{O} \rightarrow \mathrm{Nb}_{2} \mathrm{O}_{5}+10 \mathrm{H}^{+}+5 \mathrm{SO}_{4}{ }^{2-}+10 \mathrm{e} \\
& \mathrm{Nb}_{2} \mathrm{O}_{5}+6 \mathrm{HF} \rightarrow \mathrm{H}_{2} \mathrm{NbOF}_{5}+\mathrm{NbO}_{2} \mathrm{~F} \cdot 0.5 \mathrm{H}_{2} \mathrm{O}+1.5 \mathrm{H}_{2} \mathrm{O} \\
& \mathrm{NbO}_{2} \mathrm{~F} \cdot 0.5 \mathrm{H}_{2} \mathrm{O}+4 \mathrm{HF} \rightarrow \mathrm{H}_{2} \mathrm{NbOF}_{5}+1.5 \mathrm{H}_{2} \mathrm{O}
\end{aligned}
$$

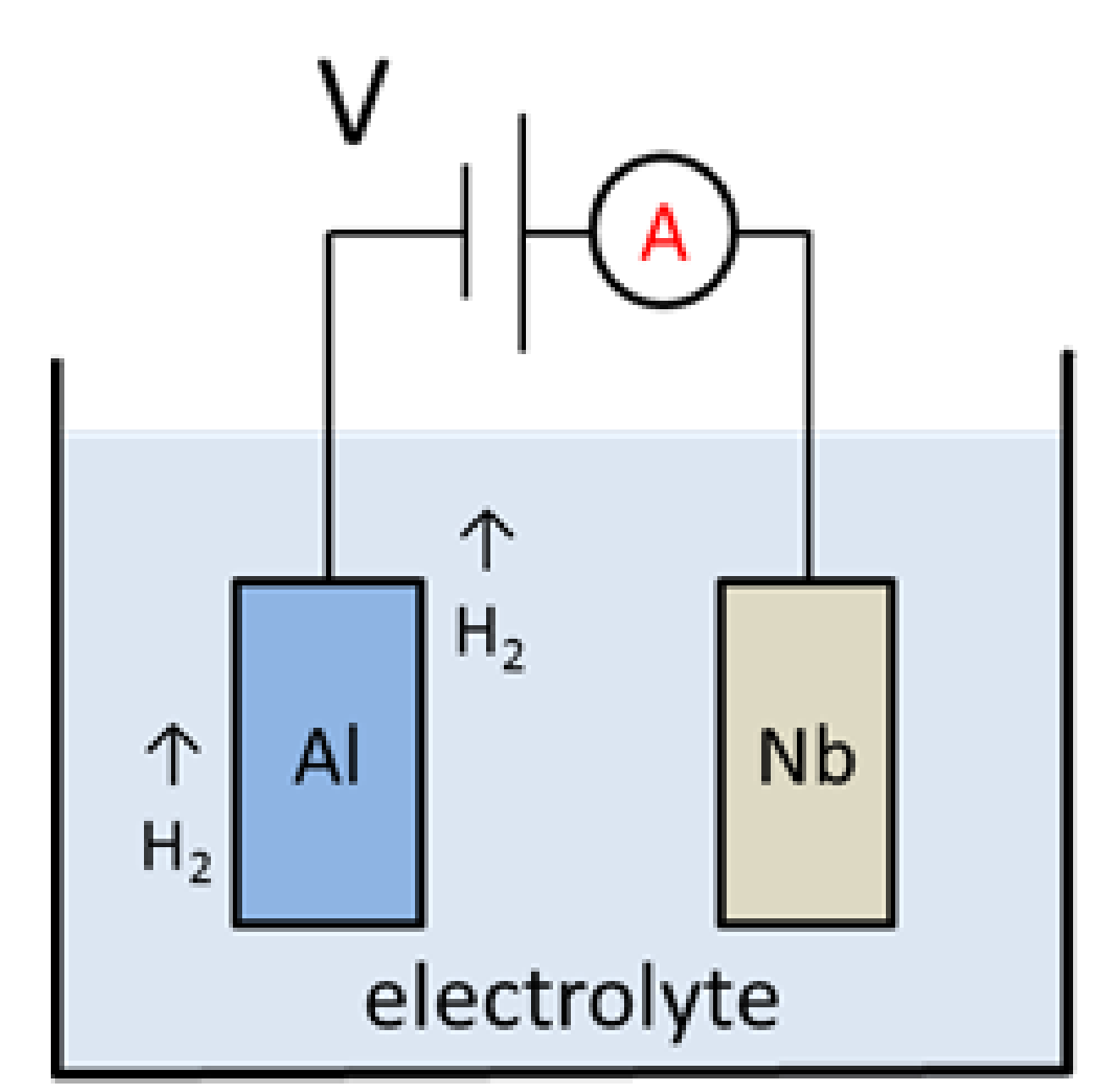

Schematic image of EP

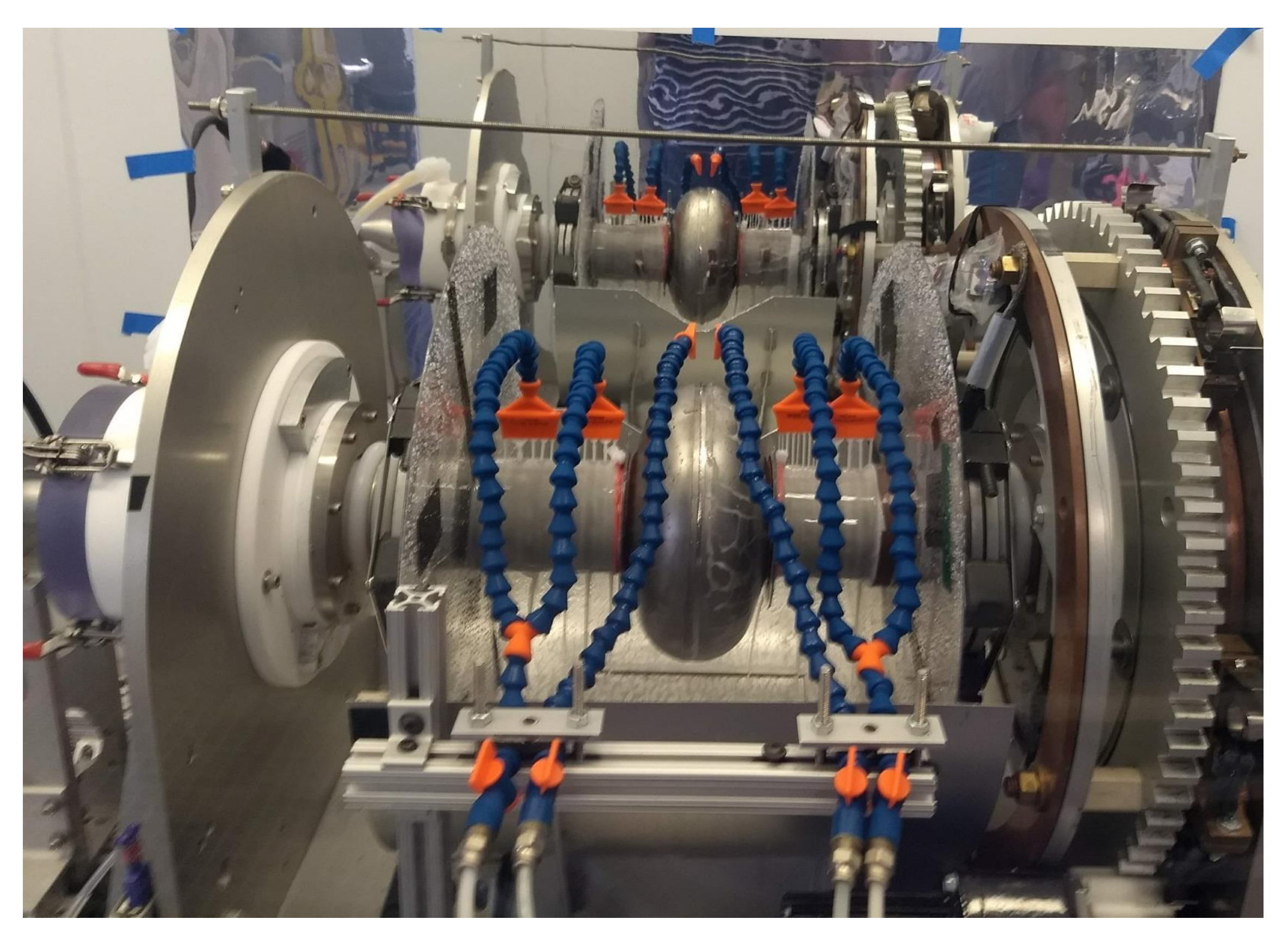

Cooling shower for outer surface This provides a precise control of EP temperature and $\mathrm{Nb}$ removal.
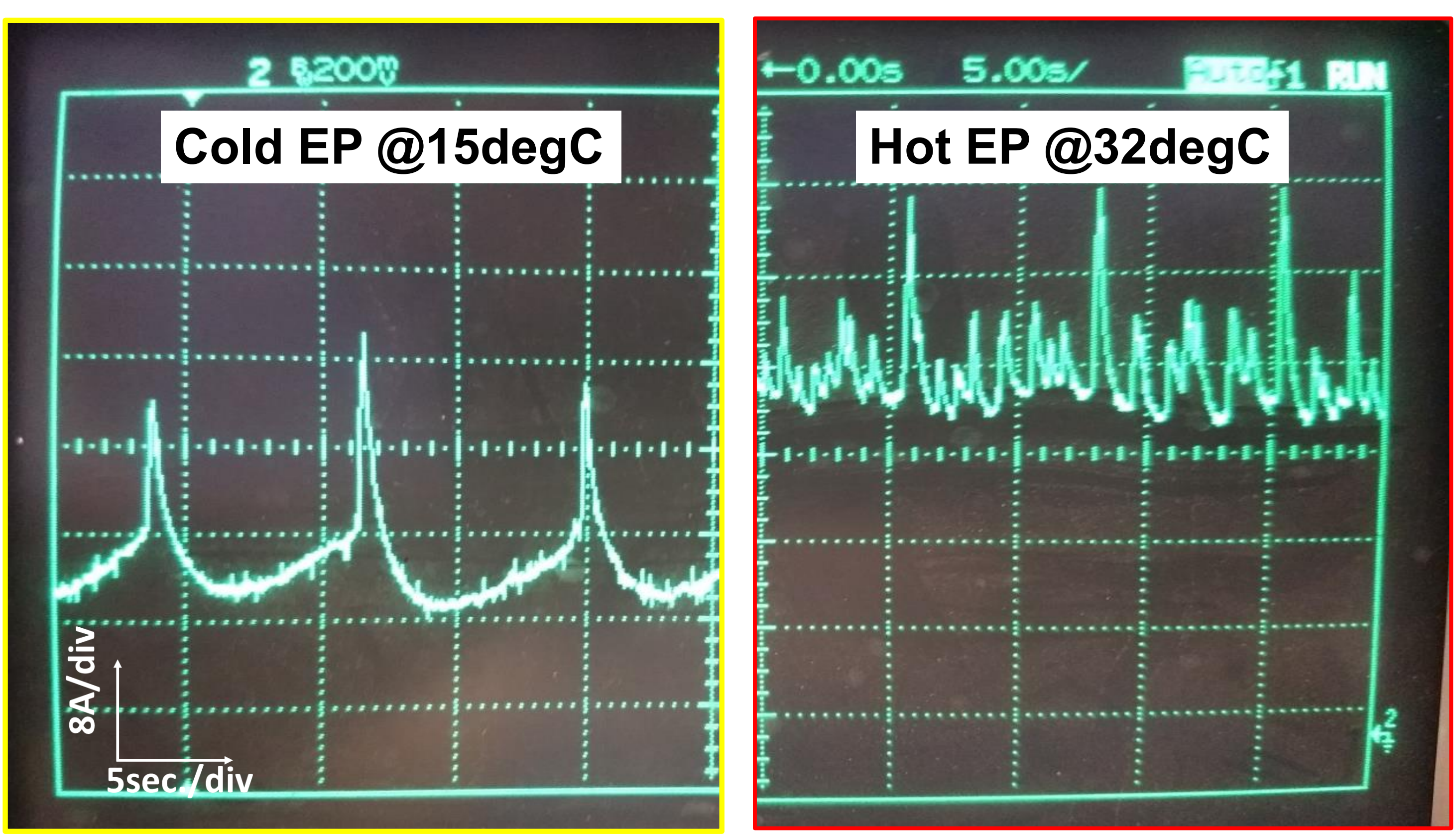

Current profiles during Cold/Hot EP process Cold EP pushes a gradient higher than std. Hot EP on N-doped cavities. R\&D on cold EP (15 2degC) is in progress to understand more details.

\begin{tabular}{lcc}
\multicolumn{1}{c}{ Parameters } & Cold EP & Hot EP \\
\hline Target removal & $10[\mu \mathrm{m}]$ or less & $>10[\mu \mathrm{m}]$ \\
EP voltage & \multicolumn{2}{c}{$18[\mathrm{~V}]$} \\
EP current & $15[\mathrm{~A}]$ & $40[\mathrm{~A}]$ \\
Equator temp. & $15[\mathrm{C}]$ & $32[\mathrm{C}]$ \\
Beam tube temp. & $0[\mathrm{C}]$ & $5[\mathrm{C}]$ \\
Acid temp. & $12[\mathrm{C}]$ or below & $20[\mathrm{C}]$ \\
Removal rate & $5[\mu \mathrm{m} /$ hour $]$ & $13[\mu \mathrm{m} / \mathrm{hour}]$ \\
Acid circulation & \multicolumn{1}{c}{$1.5 \sim 2.3[\mathrm{~L} / \mathrm{min}]}$. \\
Cavity rotation & \multicolumn{2}{c}{$1[\mathrm{revolution} / \mathrm{min}]}$. \\
Nitrogen gas flow & \multicolumn{2}{c}{$1[\mathrm{~L} / \mathrm{min}]}$. \\
\hline
\end{tabular}

Fermi EP conditions for $1.3 \mathrm{GHz}$ single cell Numbers are average values.

\section{Centrifugal Barrel Polishing (CBP), so called "tumbling"}

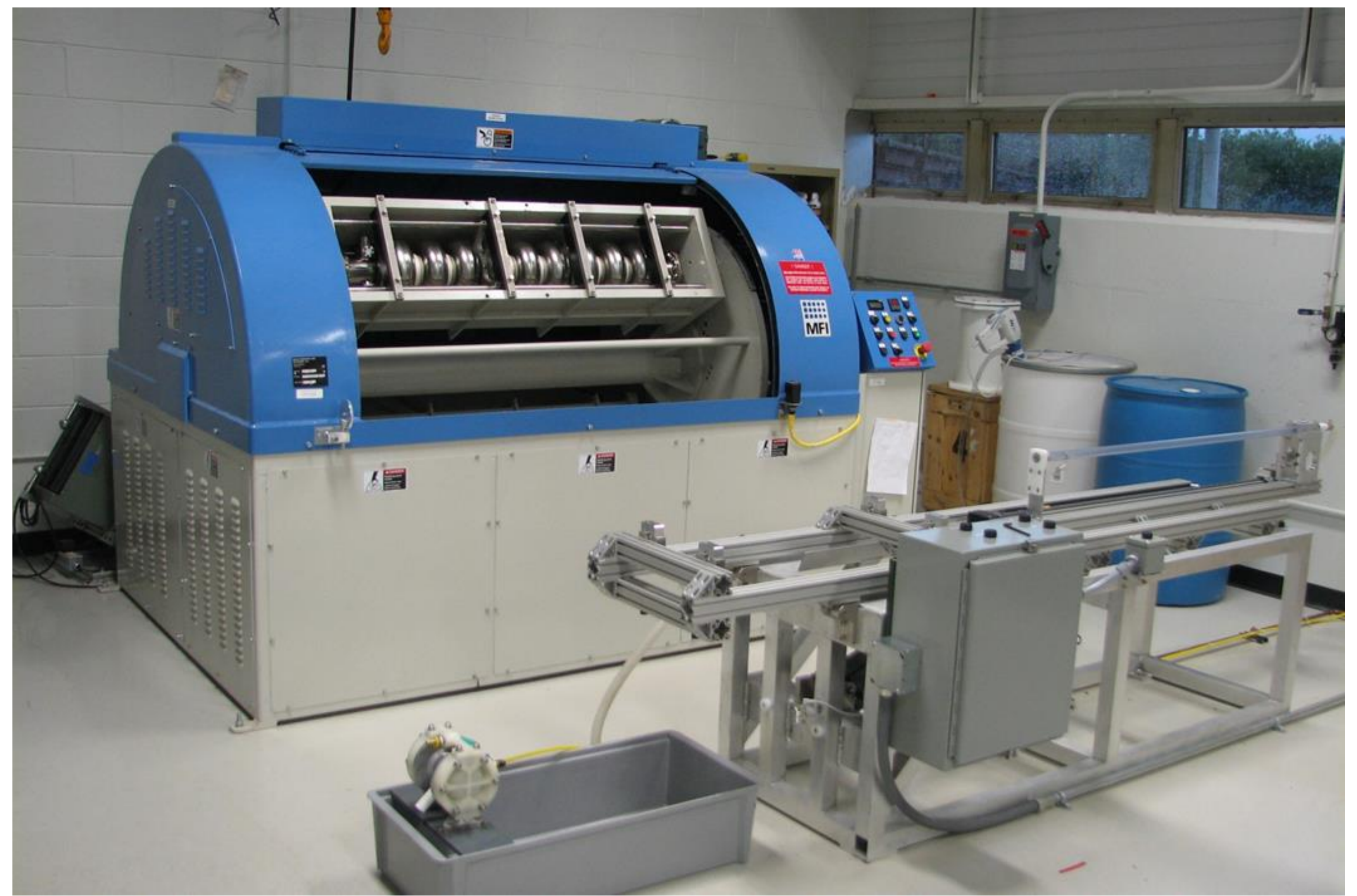

CBP tool and $1.3 \mathrm{GHz}$ 9-cell cavity in IB4 CPL This tool has two cavity containers which could hold up to one $1.3 \mathrm{GHz} 9$-cell cavity in each. The cavity rinsing tool post CBP is shown in front of CBP tool.
CBP is mechanical polishing techniques used on SRF cavities.

To perform CBP, the half volume of cavity is filled with polishing media and water.

The tool has two rotation axes: center axis and cavity axis. The cavities rotated around the center axis with the rotation speed of $\sim 100 \mathrm{rpm}$ and the cavity counter-rotated around its own axis (the cavity axis) with same rotation speed. These two rotation axes produce a strong centrifugal force on the media inside the cavity to remove material from the inner niobium surface.

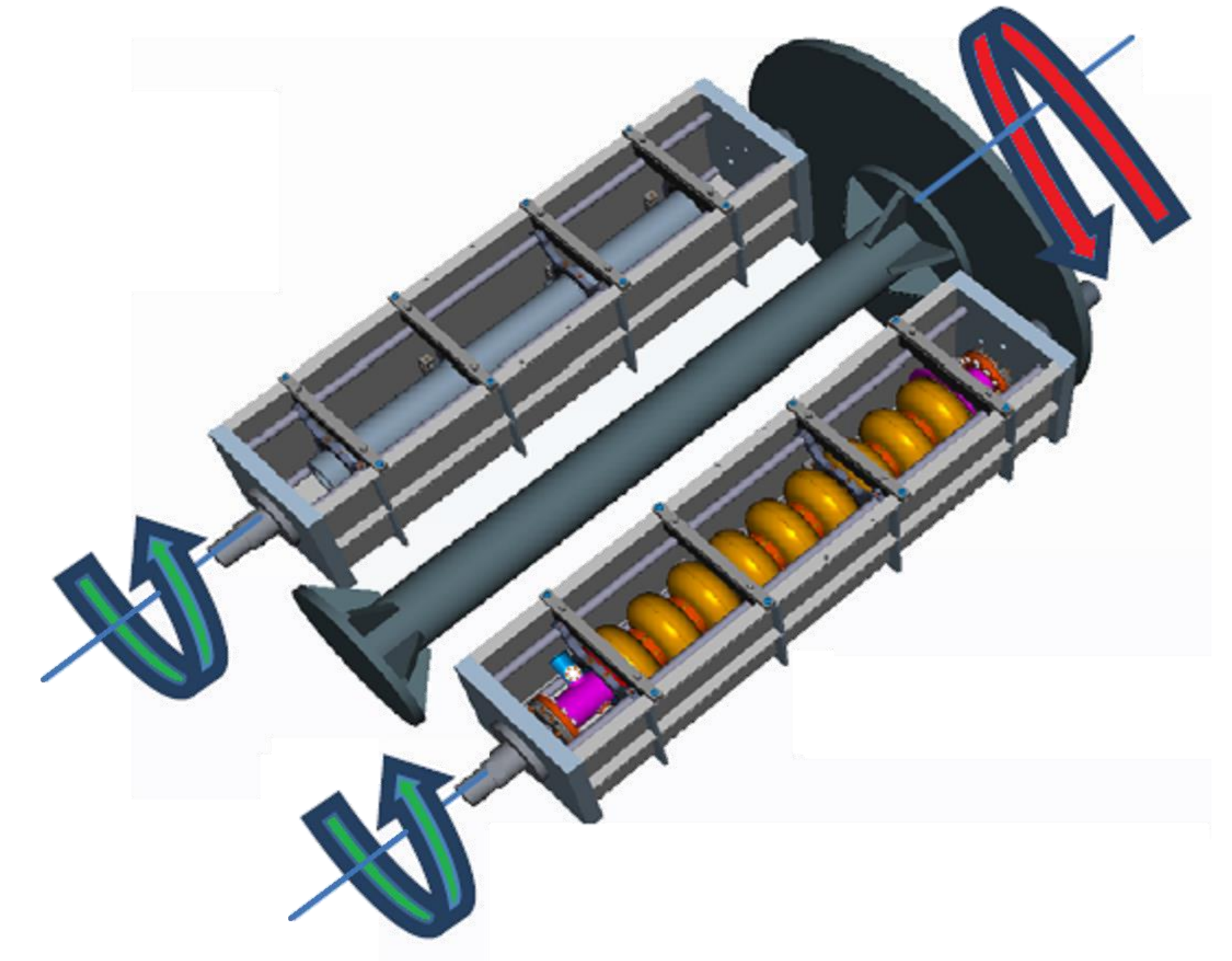

CBP is performed in several steps, rough-, intermediate-, and fine-polishing, by using different sizes, shapes, and compositions of polishing media.
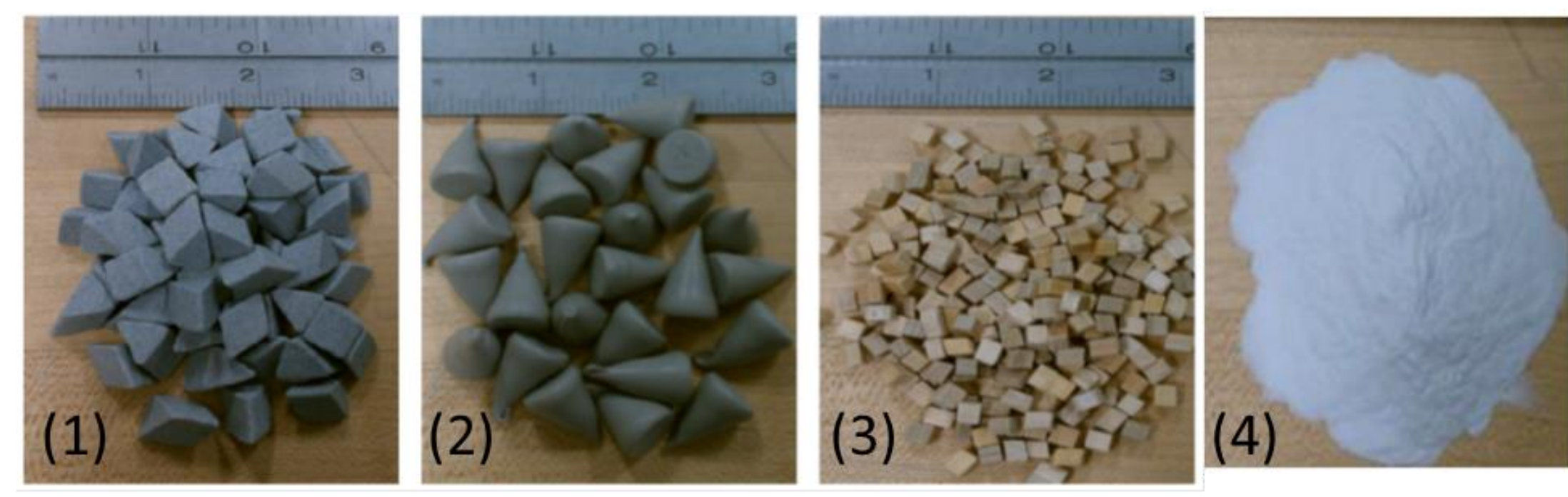

\section{Examples of CBP media}

(1) Course, K\&M ceramic; (2) Medium, RG-22 cones;

(3) hard wood blocks as carrier of (4) Al powder , size of below $18 \mu \mathrm{m})$. 